\title{
Documentation of tricuspid regurgitation by radionuclide angiocardiography
}

\author{
Fred S. Mishkin and Marvin E. Mishkin \\ From the Division of Nuclear Medicine, Department of Radiology, Martin Luther King, fr. General Hospital, \\ Los Angeles, California, U.S.A.
}

\begin{abstract}
Review of the radionuclide cardiac angiograms of 82 patients studied during the past year showed reflux of activity down the inferior vena cava in 7 , all of whom had clinical evidence for or clinical conditions associated with tricuspid regurgitation. Analysis of the flow of the radioactive bolus through the cardiac chambers demonstrates that the inferior vena cava fills only after activity enters the right ventricle and that filling is most pronounced during ventricular systole. In one case of right-sided endocarditis in which the characteristic murmur of tricuspid regurgitation was heard, this finding could not be demonstrated. These results suggest that radionuclide angiocardiography can provide a sensitive noninvasive means for documenting the presence of tricuspid regurgitation.
\end{abstract}

Although its incidence is uncertain, tricuspid regurgitation in the presence of mitral or aortic valve disease may signify serious haemodynamic abnormalities. Coexisting valvar disease responsible for functional tricuspid regurgitation or associated with an organic tricuspid lesion may make the clinical diagnosis difficult. Some have suggested that the failure to replace surgically the tricuspid valve when indicated at the time of repair of the left heart valves may result in circulatory embarrassment so severe that it precludes separation of the patient from cardiopulmonary support (Starr, Herr, and Wood, 1967). For these reasons an objective means for demonstrating the presence of tricuspid regurgitation would be welcome.

Contrast angiography uniquely provides precise anatomical detail, but catheter position through the valve and the high pressure system necessary to deliver the contrast medium may result in spurious reflux. Haemodynamic data show that with tricuspid regurgitation right atrial mean pressure exceeds superior vena caval pressure (Bloomfield et al., 1946), so that during ventricular systole there should be reversal of flow from the right atrium to the vena cava (Müller and Shillingford, 1954). Radionuclide angiocardiography is a noninvasive procedure permitting ready repetition on an outpatient basis and produces no direct haemodynamic changes (Kriss et al., 1971). Our experience suggests that this technique provides a sensitive, Received 18 March 1974. objective means for documenting the flow reversal which occurs in tricuspid regurgitation.

\section{Methods}

The records and radionuclide angiocardiograms of all 82 patients referred for the procedure during the past year were reviewed. Radionuclide angiocardiography was performed with a few modifications according to standard technique (Kriss et al., 197I). A scintillation camera positioned over the cardiac area recorded the transit of an intravenously injected radioactive bolus. A collimeter with many parallel holes projects the gamma rays onto a sensitive crystal surface coupled to an array of photomultiplier tubes which almost instantaneously detects the radioactive emissions occurring in a $28 \mathrm{~cm}$ diameter field. This is done using $X$ and $Y$ axis electronic positioning circuits. These detected events are electronically transcribed to an oscilloscope from which they may be recorded using sequential time exposures. Alternatively, the events which are analogue in nature may be converted to digital signals and stored on video tape or other digital storage devices.

Usually, parallel hole collimators with holes vertically oriented to the camera surface are used, but in order to obtain a more informative oblique view, the camera was equipped with a collimator having the parallel holes angled $20^{\circ}$ from vertical. Bringing the collimator flush against the chest with the holes angled towards the patient's right produces a $20^{\circ}$ left anterior oblique view, without the loss of photon yield which results when a straight parallel hole collimator is drawn away from the chest to place it at a $20^{\circ}$ oblique position to the patient. The radionuclide, usually $20 \mathrm{mCi}$ of ${ }^{89 \mathrm{~m}} \mathrm{Tc}$ as pertechne- 
tate or labelled serum albumin, is placed in a venotube reservoir, and then flushed as a bolus through a scalp vein needle into the external jugular system, preferred because of its lack of valves, or into a medial antecubital vein leading to the basilic vein.

The passage of the bolus is recorded along with the electrocardiogram on videotape. A complete account of this method for obtaining and storing the data is available in the work of Ashburn et al. (1973). These data are later played back and photographed onto $4 \times 5 x$-ray film during 0.5 or 1.0 sec time intervals depending upon the circulation time. Data are also played back using a gating system coupled with the electrocardiographic signal so that recording may be made only during diastole using the 50 millisecond portion of the data asso- ciated with the QRS complex, or only during systole using the 50 millisecond interval associated with the initial portion of the $T$ wave. Such data recording allows analysis and separation of those events and motions occurring during systole from those occurring during diastole (Zaret et al., 197I). The data are also played into an online computer for generation of time-activity histogram curves which may be selected from any region of the heart and lungs (Weber, dos Remedios, and Jasko, I972).

\section{Findings}

In 7 cases, activity appeared in the inferior vena cava before circulation of the bolus through the lungs

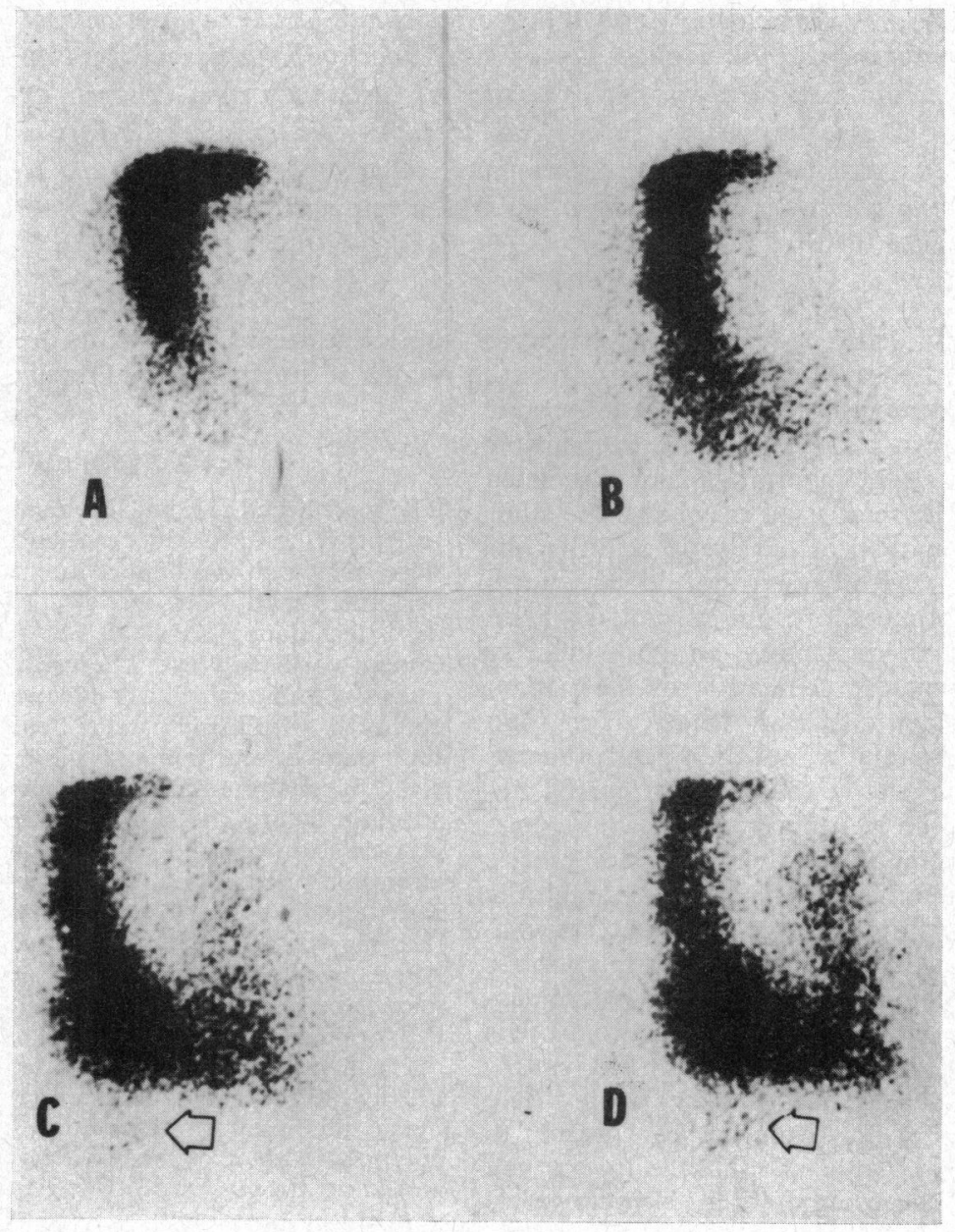

FIG. I Sequential one-second frames from the radionuclide angiocardiogram in a patient with cardiomyopathy showing regurgitation down the inferior vena cava (arrow) during right heart transit of the bolus. In $A$, the superior vena cava is filled; in $B$, the right atrium; in $C$, the right ventricle; and in $D$, the pulmonary outflow tract. 
TABLE Clinical data in patients with tricuspid regurgitation

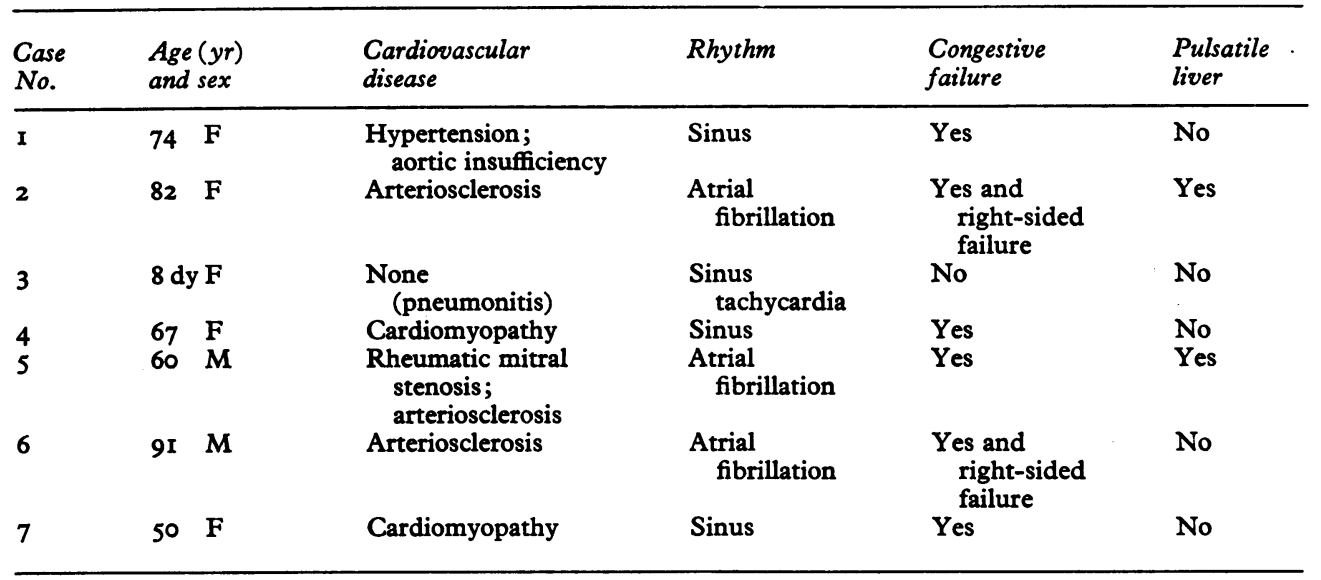

(Fig. I). Each patient either had clinically certain evidence of tricuspid regurgitation or a situation associated with functional tricuspid regurgitation caused by increased resistance to passage of the bolus across the tricuspid valve (Table). In one case studied, a drug addict with right-sided staphylococcal endocarditis, there was a systolic murmur probably accentuated during inspiration, but no reflux was demonstrated. It is not certain the patient had tricuspid regurgitation. That the activity actually represents regurgitation from the right ventricle rather than from the atrium is demonstrated by two findings. First, the activity in the vena cava does not appear until there is activity in the right ventricle. This is shown by comparing the peaks of time-activity histograms generated from the right atrium, the outflow portion of the right ventricle, and the inferior vena cava (Fig. 2). Peak activity in the vena cava occurs only after the peak in the right ventricular outflow tract. Secondly, gated images for the same period show much more activity in the inferior vena cava during ventricular

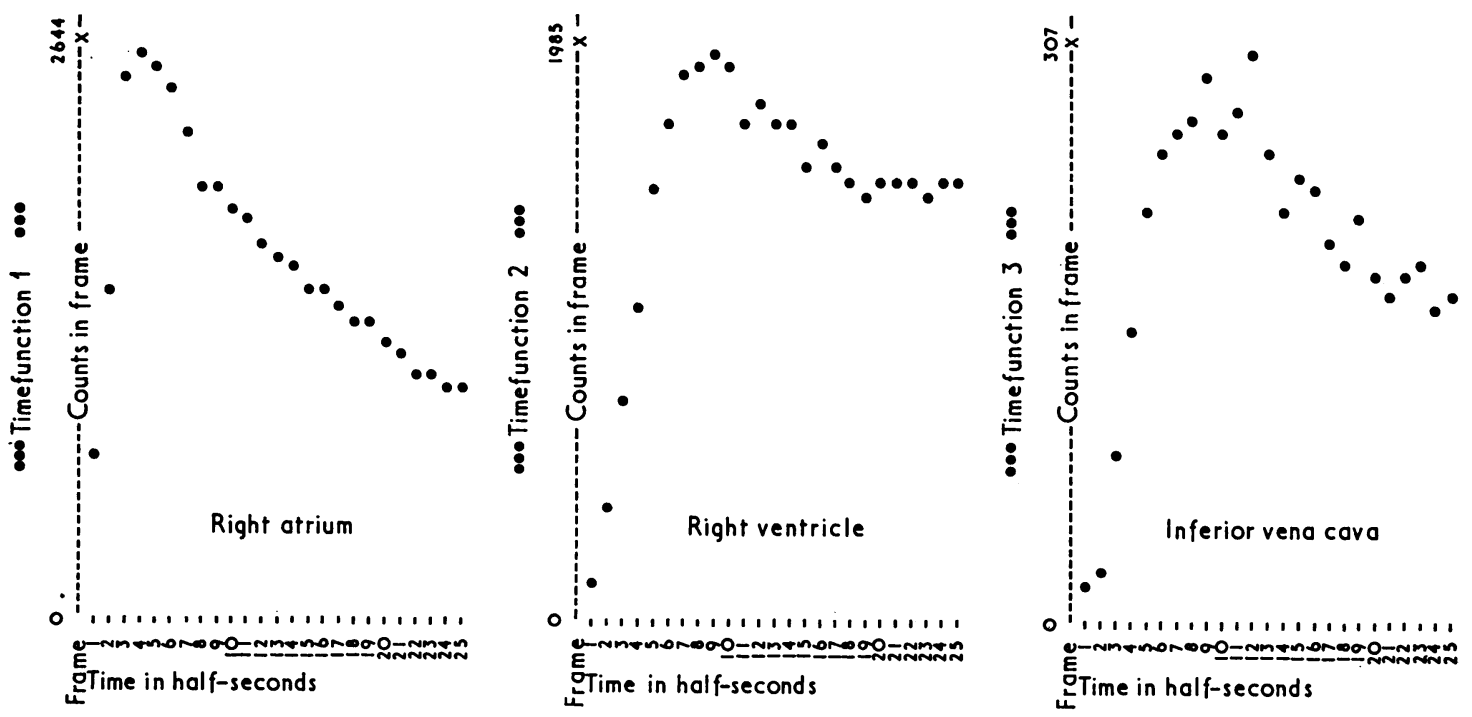

FIG. 2 Time-activity histograms generated from the right atrium, right ventricle, and inferior vena cava. Each point represents a half-second time interval. Peak activity can be seen to occur in the right atrium at 2 seconds and in the right ventricle at 4.5 seconds. A double peak is seen in the inferior vena cava, the first at 4.5 seconds, and the second and higher peak at 6 seconds. 


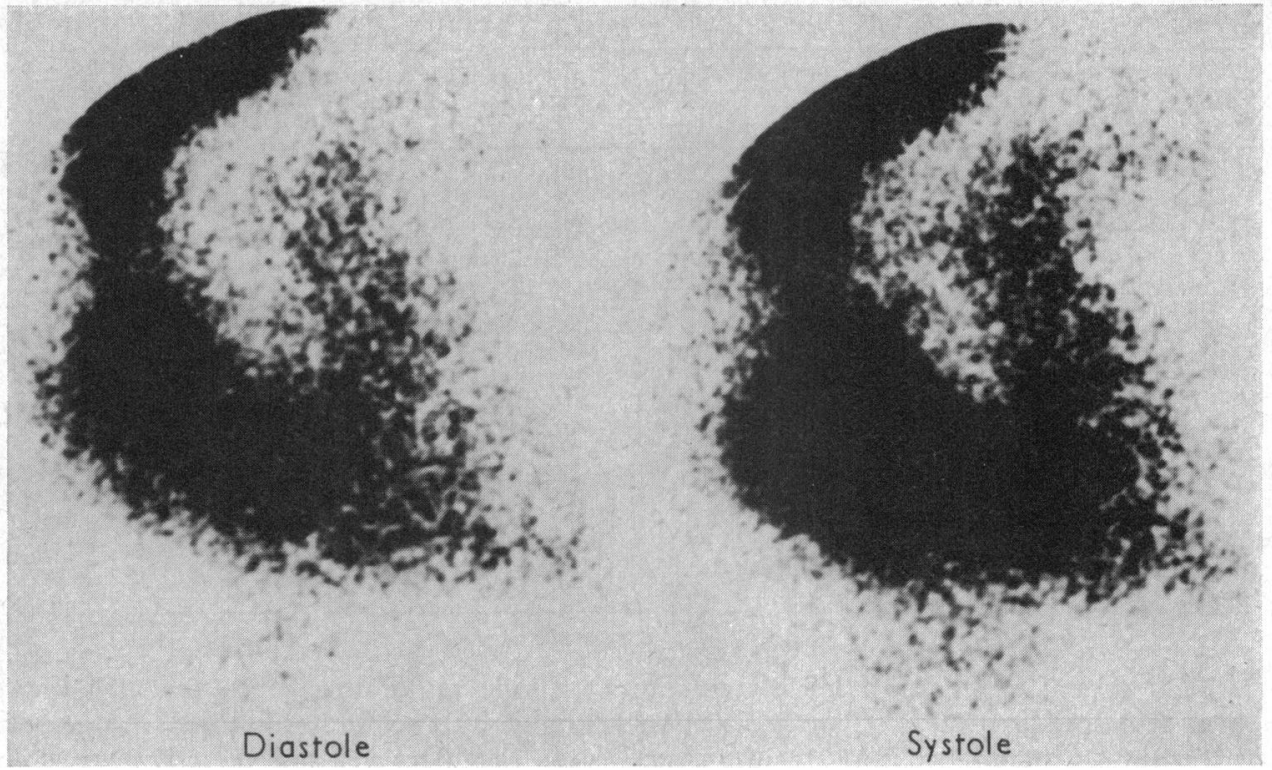

FIG. 3 Gated images during the first I2 seconds of transit of the bolus before filling of the lungs. The image during diastole is a composite of events occurring during the 50 millisecond interval associated with the $Q R S$ complex during this period, and the image during systole a summation of events occurring with the 50 millisecond interval associated with the initial portion of the $T$ wave. Regurgitation can be seen to be greatest during systole, coincident with expansion of the right atrium and contraction of the right ventricle.

systole than during diastole (Fig. 3). Systolic expansion of the right atrium may also be documented.

\section{Conclusion}

Intravenous radionuclide angiocardiography offers a simple objective means of documenting the presence of tricuspid regurgitation. A gating system or computer is not necessary to document the reflux. The sensitivity in three cases was greater than the clinical criteria, which are admittedly inadequate.

\section{References}

Ashburn, W. L., Kostuk, W. J., Karliner, J. S., Peterson, K. L., and Sobel, B. E. (1973). Left ventricular volume and ejection fraction determination by radionuclide angiography. Seminars in Nuclear Medicine, 3, 165.

Bloomfield, R. A., Lauson, H. D., Cournand, A., Breed, E. S. and Richards, D. W. (1946). Recording of right heart pressures in normal subjects and in patients with chronic pulmonary disease and various types of cardiocirculatory disease. Fournal of Clinical Investigation, 25, 639.

Kriss, J. P., Enright, L. P., Hayden, W. G., Wexler, L, and Shumway, N. E. (197I). Radioisotope angiocardiography: Wide scope of applicability in diagnosis and evaluation of therapy in diseases of the heart and great vessels. Circulation, 43, 792.

Müller, O., and Shillingford, J. (1954). Tricuspid incompetence. British Heart fournal, 16, 195.

Starr, A., Herr, R. H., and Wood, J. A. (1967). Mitral replacement. Review of six years' experience. Fournal of Thoracic and Cardiovascular Surgery, 54, 333.

Weber, P. M., dos Remedios, L. V., and Jasko, I. A. (1972). Quantitative radioisotopic angiocardiography. Fournal of Nuclear Medicine, 13, 815.

Zaret, B. L., Strauss, H. W., Hurley, P. J., Natarajan, T. K., and Pitt, B. (1971). A noninvasive scintiphotographic method for detecting regional ventricular dysfunction in man. New England fournal of Medicine, 284, I165.

Requests for reprints to Dr. F. S. Mishkin, Martin Luther King Jr. General Hospital, I202I South Wilmington Avenue, Los Angeles, California 90059, U.S.A. 